\title{
Study Design Description
}

National Cancer Institute

\section{Source}

National Cancer Institute. Study Design Description. NCI Thesaurus. Code C147139.

A summary of the components of study design employed for the study including, but not limited to, interventions, study populations, blinding, treatment assignment methods, etc. 